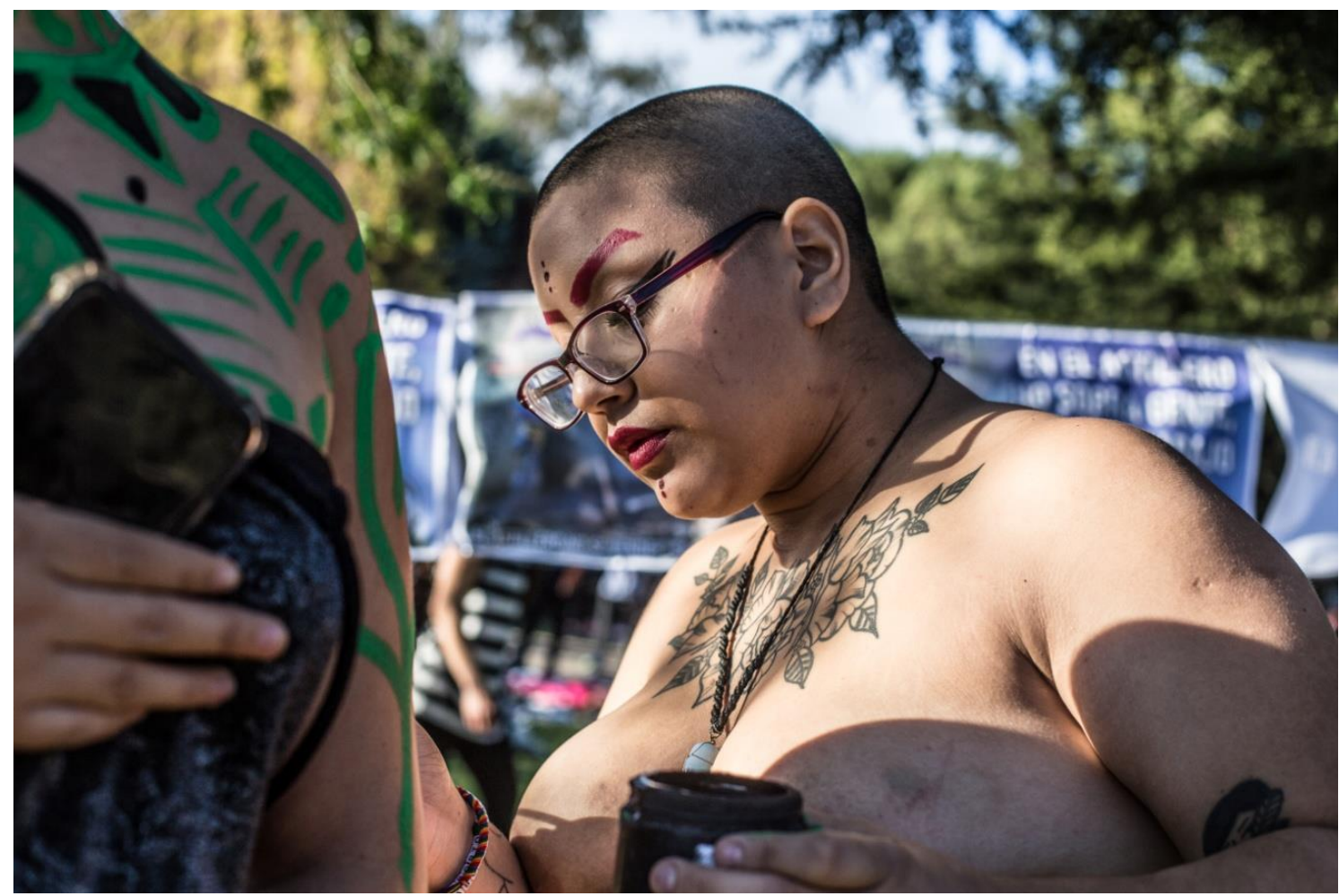

FOTOS: Irina Morán - Revista Alfilo - Periodista Feminista. Militante de Ni Una Menos Córdoba y Mujeres por un parto Respetado 


\title{
PEDAGOGÍAS FEMINISTAS: ESTRATEGIAS UNA EDUCACIÓN EMANCIPADORA Y DECOLONIAL
}

\author{
Irene Martínez Martín ${ }^{1}$
}

\begin{abstract}
Resumen: ¿Qué lugar ocupa la educación en la reproducción de desigualdades de género? ¿Nuestras prácticas educadoras transforman los contextos de desigualdad y violencia? ¿Es posible impulsar una educación transgresora del sistema dominante patriarcal? Este artículo busca construir respuestas en torno a las pedagogías feministas. Se realiza un estudio bibliográfico descriptivo y reflexivo que toma como referencia los diversos espacios de acción del feminismo y la educación. Este artículo pone en diálogo perspectivas epistemológicas diversas, buscando establecer conexiones entre: feminismos, pedagogía, diversidad y cultura. De esta manera, se concluye con la propuesta de unas líneas estratégicas que, por un lado interpelan a los agentes educativos en su toma de posición ante las desigualdad de género y, por el otro, construye acciones para subvertir las prácticas y teorías educativas tradicionales prestando especial atención a la interseccionalidad, las diversidades, los lenguajes, los curriculums y la pedagogía crítica.

Palabras-clave: Pedagogía. Feminismos. Interseccional. Decolonial. Emancipadora.
\end{abstract}

\section{FEMINIST PEDAGOGIES: STRATEGIES FOR AN EMANCIPATING AND DECOLONIAL EDUCATION}

\begin{abstract}
What place does education in the gender inequalities reproduction? Do our educational practices making changes in the inequality and violence contexts? Is it possible to promote a transgressive education of the patriarchal dominant system? This paper asks answers around feminist pedagogies. A descriptive and reflective bibliographical study with a feminist and educational approach is done at the research process. This paper puts forward a dialogue between different epistemological perspectives about: feminisms, pedagogy, diversity and culture. In this way, it conclude with a strategic lines that; On the one hand, it challenges educational agents in their position on gender inequality; and, on the other hand, it makes action strategies to subvert the traditional educational practices and theories about: intersectionality, diversity, languages, curricula and critical pedagogy.
\end{abstract}

Keyword: Pedagogy. Feminism. Intersectional. Decolonial. Emancipating.

\section{INTRODUCCIÓN}

Empezamos este texto con un relato breve de Eduardo Galeano, de su libro "Mujeres":

\section{JUANA}

Como Teresa de Ávila, Juana Inés de la Cruz se hizo monja para evitar la jaula del matrimonio. Pero también en el convento su talento ofendía. ¿Tenía cerebro de hombre esta cabeza de mujer? ¿Por qué escribía con letra de hombre? ¿Para qué quería pensar, si guisaba tan bien? Y ella, burlona, respondía:

— ¿Qué podemos saber las mujeres, sino filosofías de cocina?

Como Teresa, Juana escribía, aunque ya el sacerdote Gaspar de Astete había advertido que a la doncella cristiana no le es necesario saber escribir, y le puede ser dañoso.

Como Teresa, Juana no sólo escribía, sino que, para más escándalo, escribía indudablemente bien.

En siglos diferentes, y en diferentes orillas de la misma mar, Juana, la

\footnotetext{
${ }^{1}$ Universidad Complutense de Madrid, Departamento Estudios Educativos. imarti02@ucm.es Momento: diálogos em educação, E-ISSN 2316-3100, v. 28, n. 3, p. 350-365, set./dez, 2018.
} 
mexicana, y Teresa, la española, defendían por hablado y por escrito a la despreciada mitad del mundo.

Como Teresa, Juana fue amenazada por la Inquisición. Y la Iglesia, su Iglesia, la persiguió, por cantar a lo humano tanto o más que a lo divino, y por obedecer poco y preguntar demasiado.

Con sangre, y no con tinta, Juana firmó su arrepentimiento. Y juró por siempre silencio. Y muda murió.

¿Qué contextos habitamos? ¿Cómo los habitamos? ¿Cómo reaccionamos ante las desigualdades? ¿Con nuestras acciones reproducimos o transformamos nuestros espacios? ¿Es la educación un motor de emancipación y liberación ante las opresiones? ¿Qué implica despatriarcalizar y descolonizar la educación, como indica María Galindo (2014)? Estas preguntas, y otras muchas, nos invitan a la reflexión en torno a cómo se construye el mundo que vivimos y a cómo se normaliza una estructura social determinada.

En este texto, y en este dossier temático, dedicado a la educación feminista como respuesta a los desafíos de la sociedad actual vamos a explorar precisamente esto: cómo desde la educación y los feminismos podemos dar respuesta a esos interrogantes.

\section{LOS ESPACIOS QUE VIVIMOS}

Empezamos nuestra reflexión mirando, escuchando y cuestionando las realidades que habitamos. Una de las cuestiones que los movimientos feministas proponen, ya desde sus reivindicaciones históricas, es la necesaria toma de conciencia crítica en torno a la construcción del mundo desde cánones (hetero) patriarcales. Es decir, la normalización de unas estructuras sociales basadas en el sistema sexo-género tradicional (Rubin, 1975) donde lo estereotipado como masculino-dominante se le otorga un estatus de poder y superioridad sobre todo aquello categorizado como "los otros", "las otras", "les otres".

En estos términos hablamos de un mundo dicotomizado y jerarquizado (Kate Millet, 1995) donde lo masculino, occidental, heterosexual, urbano, con educación superior, jóven, etc. se considera normativo y lleno de privilegios. Kimberley Crenshaw (1994) denominará a este cruce de opresiones y privilegios, interseccionalidad del género con la clase social, la etnia, la cultura, la orientación sexual, la edad, etc.

Los movimientos feministas se hacen eco de la evolución del concepto sexogénero más allá de esta estructura dicotomizada. Así, tanto el género como el sexo, se Momento: diálogos em educação, E-ISSN 2316-3100, v. 28, n. 3, p. 350-365, set./dez, 2018. 
consideran conceptos construidos culturalmente (Butler, 2001) otorgándole especial atención a la construcción de identidades de género más allá de la oposición hombre "vs" mujer haciendo del género y del sexo conceptos fluidos, en construcción y abiertos al cambio.

Esta concepción postestructuralista y abierta es fundamental para entender el posicionamiento de la educación feminista como elemento transformador e impulsor de categorías de género dinámicas.

Las pedagogías feministas toman como punto de partida la visibilización de las estructuras patriarcales que influyen en todas las dimensiones del desarrollo de las personas, haciendo referencia a: lo individual, lo político, lo económico, lo laboral, lo familiar, el ocio, los cuerpos, lo sexual, lo afectivo, lo público, los medios de comunicación, etc.

Volvemos a interrogarnos: ¿Qué vemos?, o más específicamente, ¿a quién vemos y a quién no vemos? No vemos cuerpos diversos, racializados, no vemos mujeres con éxito, nos vemos hombres cuidadores... vemos únicamente un discurso normalizado y estereotipado como racista, homófobo, machista y clasista.

En este proceso de toma de conciencia nos cuestionamos acerca de quiénes vemos, pero también, acerca de cómo se organiza la estructura productiva "vs" reproductiva de nuestro sistema político-económico. Desde la economía feminista se habla de la división sexual del trabajo, los techos de cristal, la brecha de género en salarios, la feminización de ciertas profesiones (peor remuneradas), el acoso sexual, la imposición de valores como la competitividad, el control de los recursos, entre otros aspectos. Encontramos, entonces, una organización socio-económica donde los valores productivos capitalistas están en manos de hombres (una vez más normativos, blancos, clase media-alta...) entrando en contradicción con los valores de reproducción y cuidado asociado a otro modelo de desarrollo. Nos preguntamos, entonces: ¿quién dedica más tiempo a las tareas del hogar? ¿sobre qué personas recaen las tareas del cuidado? ¿quién goza de menor tiempo libre y de descanso?

Amaia Pérez y Sira del Río (2002) señalan que "habíamos salido de la sartén para caernos en el cazo salarial (con la sartén incluida). Y ya en el cazo laboral (discriminadas y en muchos casos precarias) las mujeres tuvimos que seguir haciendo el trabajo "de la casa" porque se entendía que era un asunto "nuestro". 
Las pedagogías feministas valoran la ética de cuidado como un elemento fundamental de sus propuestas. El trabajo doméstico, de cuidados y sostenedores de vida, tienen un impacto fundamental en el mantenimiento de la sociedad capitalista y patriarcal. Silvia Federicci (2013) profundiza en esta idea señalando cómo las mujeres hemos sido socializadas (y convencidas) de que las tareas del cuidado son inherentes a nosotras pero sin aclarar que hay un único beneficiario: el capital.

Además, en este análisis cabe introducir la variable de la interseccionalidad para tomar conciencia de cómo las tareas del cuidado y domésticas tienen un fuerte componente de clase y etnia, por ejemplo, entre mujeres migrantes o de clases desfavorecidas. Amaia Orozco (2007) nos habla de las cadenas globales de cuidados desde dimensiones transnacionales y bajo unos ejes de poder, de clase, etnia y género.

Aunque el análisis de los contextos que habitamos desde la mirada feminista puede ser más extenso fijándonos, por ejemplo, en la salud, la participación, el liderazgo, los derechos y las leyes, los puestos de poder, la educación... vamos terminar haciendo referencia a las violencias machistas y hacia las mujeres.

El análisis hecho hasta el momento tiene como resultado un sistema desigual que ejerce violencia hacia todas esas personas que no entran dentro de los límites de lo normativo. Se incluyen múltiples formas de violencia: física, psicológica, sexual, ambiental, económica, simbólica, social, institucional, familiar, etc. La expresión de esta violencia, además, varía siendo más o menos explícita o visible según los contextos.

Estamos entonces ante un sistema patriarcal que pone de manifiesto la dominación masculina sobre la sociedad en general y las mujeres en particular. Es un sistema de organización social, político, económico y cultural que promueve una relación asimétrica y jerarquizada. Este sistema intersecciona con otros sistemas de opresiones que son racistas, clasistas, LGTBQfóbicos, entre otros. Es decir, un sistema que socializa y normaliza diferentes sistemas de opresión y violencias.

Todas estas dimensiones conforman los núcleos de socialización y aprendizaje cultural, que desde la infancia, van normalizando un mundo dicotomizado y jerarquizado. John Dewey (1970) reclama una educación para la ciudadanía democrática, es decir, una educación que que convierta los procesos de enseñanza y aprendizaje en espacios de vida, de ensayo y de pensamiento crítico favoreciendo la Momento: diálogos em educação, E-ISSN 2316-3100, v. 28, n. 3, p. 350-365, set./dez, 2018. 
toma de conciencia y de pertenencia responsable a una sociedad donde si hay privilegios, hay privación de los mismos y, por lo tanto, desigualdades y opresiones.

\section{REPRODUCIR O TRANSFORMAR}

Hemos visto cómo desde las pedagogías feministas necesitamos una toma de conciencia de los mundos que habitamos, pero este proceso no puede entenderse al margen de una toma de conciencia individual y de cómo actúan los procesos de socialización diferenciada.

Sabemos (Irene Martínez y Gema Ramírez, 2017) que desde el nacimiento se nos otorgan una serie de atributos según nuestro sexo biológico y, que esta primera categorización social, va a ir marcando una serie de estereotipos y roles de cómo debemos ser y qué debemos hacer en función de si hemos sido asignados como hombres o como mujeres. Para garantizar que estos imaginarios permanezcan inalterables existe un complejo régimen de aprendizaje y de sanciones para hacer cumplir estos mandatos.

Marcela Lagarde (1996) habla de una configuración de reglas básicas que ayudan a que cuando un poder aumenta, directamente se resta poder de otro, es decir, la relación jerárquica se construye por oposición a una subordinación jerárquica y los valores que se universalizan en base a esta división.

Las pedagogías feministas, entonces, buscan romper con estos procesos de socialización diferenciada que, al considerarse cultural, varían de unos contextos a otros pero que comparten en su base la idea de ser un proceso de aprendizaje que dura toda la vida y a través del cual las personas vamos interiorizando lo propio de nuestro sexogénero (roles y estereotipos).

En este contexto se entiende el feminismo y la educación como un movimiento social y teórico-práctico de denuncia, de resistencia y de transformación (Nuria Varela, 2003). Debe ser considerado un proceso dinámico, creativo y abierto al análisis crítico. Así, no hablamos de herramientas o de teorías cerradas, sino que incluimos variables multidimensionales desde lo político, lo económico, lo social, lo cultural y lo personal. Busca denunciar la reproducción de desigualdades para promover la transformación en todas las esferas que generan esas desigualdades.

Educación y feminismos no es un único marco o teoría, es una diversidad de posicionamientos, acciones, luchas, demandas... que tienen como propuesta: ser radical, ir a la raíz de las desigualdades; incluir la multidimensionalidad; ser transversal, Momento: diálogos em educação, E-ISSN 2316-3100, v. 28, n. 3, p. 350-365, set./dez, 2018. 
horizontal, autónomo, autogestionado y sostenible; apostar por la creatividad; construir desde la crítica; promover acciones subversivas; motivar redes de sororidad y comunitarias; incluir un enfoque decolonial e interseccional; y educar en las diversidades.

\section{EDUCACIÓN COMO PRÁCTICA DE LIBERTADES}

En estaecamino de construcción de pedagogías feministas cabe posicionarse en una educación crítica, emancipadora y transgresora. En palabras de Paulo Freire (1979) la educación no es la mera transmisión de conocimientos impuestos, sino la posibilidad de crear espacios de pensamiento, diálogo y acción.

Las enseñanzas de la pedagogía crítica nos hablan de la educación como elemento no neutro, es decir, con un fuerte componente político y responsable de la reproducción de aquellos imaginarios imperantes en cada contexto social. A través de la educación podemos reproducir privilegios y desigualdades o bien, provocar las condiciones para su transformación.

Preguntarnos qué, cómo, a quién, para qué... educamos nos invita a reflexionar acerca del posicionamiento teórico-práctico que guía nuestra acción educadora. Así, siguiendo a Van der Vleuten (1996) las pedagogías feministas son emancipadoras y críticas. Invitan a la construcción del conocimiento desde una concepción abierta y diversa del mismo donde no existe un saber mejor que otro, si no múltiples formas de conocer la realidad. Una educación centrada en los y las sujetos que aprenden estableciendo dinámicas horizontales y relaciones basadas en el amor, el cuidado y el respeto por el/la educando. Todas las personas sabemos y tenemos experiencias válidas, esto debe ser el inicio de cualquier experiencia de aprendizaje. El rol de la educadora/o feminista es activar el aprendizaje, guiar, cuestionar, dialogar, fomentar el descubrimiento más allá de la mera transmisión de saberes cerrados. La educación es flexible, dinámica y creativa. Los objetivos, contenidos, metodologías de aprendizaje y evaluación son formativos, críticos y al servicio de quien aprende, siendo individualizados y valorando las diferencias; así como el aprendizaje es colectivo y colaborativo. No se da una única fuente de conocimiento ni un único canal de comunicación, hay diversidad de recursos y de elementos comunicadores. Se piensa más allá de la integración, incluimos y aprendemos de y para un mundo de diferencias.

Así, las pedagogías feministas se construyen desde varias alternativas, siendo: feministas, descoloniales y críticas. Siguiendo a Irene Martínez (2016) algunas líneas Momento: diálogos em educação, E-ISSN 2316-3100, v. 28, n. 3, p. 350-365, set./dez, 2018. 
reflexivas que debemos tener en cuenta para construir estas pedagogías son:

a) interseccionalidad de los sistemas de opresión y privilegios

b) toma de conciencia del lugar que ocupamos y los contextos que habitamos

c) visibilización de las diferencias y las diversidades

d) transversalización de masculinidades y feminidades no hegemónicas

e) aprendizaje de la genealogía e historia de las mujeres y colectivos no normativos

f) ampliación de tiempos y espacios de la educación más allá de los curriculum oficiales

g) impulso de metodologías desde las resistencias y la participación activa, cooperativa, comunitaria y popular.

h) inclusión de la perspectiva feminista en la ética de los cuidados como revolución de las bases educativas institucionalizadas

i) formación del profesorado de calidad y transgresora

j) creación de redes y dinámicas de encuentro

k) educación para una ciudadanía contra-hegemónica

1) feminismo radical, centrado en la base y estructuras de las desigualdades

m) educación para la paz, la no violencia y la transformación del conflicto desde una construcción social crítica.

\section{PEDAGOGÍA DECOLONIAL Y DESDE LAS INTERSECCIONALIDADES}

¿Qué implicaciones tiene pensar la educación desde los términos de interseccionalidad y decolonialidad? ¿Cómo avanzar en la des-construcción dicotómica del mundo? ¿Cómo convertir los procesos necesarios de (des-) aprendizaje en momentos de transformación? ¿Cómo sumar las narrativas desde los márgenes a las prácticas educativas?

Es necesario desaprender (nos) - en lo individual y lo colectivo - para poder pensar decolonialmente conjugando saberes populares con conocimientos científicos y el papel de las experiencias prácticas. En esta línea, las pedagogías feministas proponen problematizar cuestiones como: las relaciones de poder en oposición a las relaciones de cuidado; la invisibilización de las violencias evidentes, e incluso aquellas más sutiles; el protagonismo de las diversidades de sujetos, cuerpos, identidades y sexualidades; la presencia de discursos alternativos frente a los normativos; y la creación de redes de Momento: diálogos em educação, E-ISSN 2316-3100, v. 28, n. 3, p. 350-365, set./dez, 2018. 
sororidad frente a los valores individualistas y competitivos.

Pensar y hacer decolonialmente supone dar cabida a las diversidades, apostar por un diálogo constructivista, concienciar acerca del entendimiento de la complejidad social y el rol de la educación en ello, así como acerca de los procesos de aculturación que suceden cuando pensamos a los y las "otras" desde posiciones de poder y privilegios.

Para Dussel (1999) la descolonización consiste en un reconocimiento de los saberes diversos más allá de las fronteras occidentales. Incluyendo una perspectiva feminista propone dar un giro epistémico para teorizar la compleja trama de privilegios y opresiones. Lo decolonial debe ir acompañado de un proceso anti-racista, despatriarcalizador, de clase, es decir, interseccional.

Mohanty (2008) propone, en esta línea, romper con la visión unidireccional de los “ojos de occidente. El problema es complejo, Mignolo (2011), ya que una determinada manera de construir la realidad - occidental, machista, clasista, homófoba y racista - invisibiliza tanto las injusticias estructurales, como sus causas y síntomas, desprecia las consecuencias y obvia el análisis interseccional que supone incluir la diversidad de voces, protagonistas, contextos y dimensiones. Es decir, se sigue sustentando y reproduciendo una visión del mundo parcial que invisibiliza la diversidad y experiencias fronterizas.

Walsh (2013) señala que lo pedagógico y decolonial adquieren sentido desde planos políticos, sociales, culturales, económicos, familiares, emocionales, etc. como acto subversivo y de denuncia ante los binarismos impuestos desde la racionalidad globalizada, positivista, científica-técnica y racionalista que nos obliga a entender y organizar el mundo desde unas categorías jerarquizadas y patrones de poder.

Cuando exponemos esta idea de pedagogía feminista, interseccional y crítica vamos más allá de los límites de una escuela, pensamos en una educación al servicio de quienes aprenden entendida dentro, por y para las luchas sociales de liberación (Freire, 1969).

Venimos señalando la necesidad de pensar y hacer una educación liberadora y transformadora. La pedagogía feminista son estrategias de acción y metodologías que se entretejen construyendo resistencias e insurgencias. Es abrir las mentes, cuestionar prácticas, espacios y tiempos de educación. Despertar las masas haciendo educación política y social. Es desaprender las imposiciones globalizadas en nuestros cuerpos, culturas, sistemas políticos y económicos, familiares, sistema de producción, Momento: diálogos em educação, E-ISSN 2316-3100, v. 28, n. 3, p. 350-365, set./dez, 2018. 
emociones, etc.

Las líneas de acción desde un posicionamiento interseccional en las pedagogías feministas se pueden sistematizar en (Martínez y Ramírez, 2017):

- construcción del género más allá de las imposiciones occidentales jerarquizadas

- acción política crítica para resignificar los conceptos didácticos y educativos desde el feminismo decolonial

- cruce de opresiones y privilegios evitando visiones fragmentadas

- análisis de las desigualdades estructurales y sistémicas

- reconocimiento de la otredad

- deconstrucción de roles y estereotipos de género normativos

- construcción de sororidad feminista

- visibilización de las imposiciones y consecuencias del neocolonialismo en ámbitos como la salud, el medio ambiente, la educación, las relaciones sociales y la cultura.

- conocimiento y saberes feministas desde las bases sociales uniendo la acción comunitaria y los movimientos participativos

- atención, respeto y presencia de diversidades rompiendo con la idea de un sujeto normativo como elemento central del aprendizaje.

\section{ESTRATEGIAS PARA CONSTRUIR PEDAGOGÍAS FEMINISTAS}

Es interesante remarcar que las pedagogías feministas no son un receta cerrada ni una serie de pautas a aplicar ciegamente. Al contrario, la educación feminista es abierta, dinámica, reflexiva y crítica. Por esta razón es difícil hablar de un método de pedagogía feminista, más bien encontramos diversos elementos que nos ayudan a construir una educación transgresora y emancipadora. Es por ello que nos encontramos en permanente construcción.

En este marco es relevante situarnos en una apuesta por la diversidad de acceso y creación de conocimiento. Los sujetos y objetos de aprendizaje son múltiples y dinámicos existiendo variedad de realidades que pueden ser percibidas, comunicadas, aprendidas... desde muy diversas perspectivas.

Hemos contextualizado la necesidad de una pedagogía feminista, crítica y decolonial como respuesta a un mundo desigual y como transformación de una educación tradicional reproductora de los imaginarios que sustentan dichas Momento: diálogos em educação, E-ISSN 2316-3100, v. 28, n. 3, p. 350-365, set./dez, 2018. 
desigualdades.

Ahora bien, nos preguntamos: ¿cómo hacemos, en nuestro cotidiano, educación feminista? ¿En qué aspectos podemos concretar la educación feminista? En definitiva, ¿qué estrategias podemos utilizar para construir pedagogías feministas?:

- espacios: podemos tener en cuenta aspectos físicos como la organización de una sala, la decoración, la luz, el organigrama, los recursos... pero también aspectos culturales y de poder relacionados con la presencia de diversidades (mujeres, personas racializadas, con discapacidad, etc.), procesos de toma de decisiones, liderazgos, clima social de un centro, sistemas de resolución de conflictos, entre otros. La teoría educativa nos indica que el espacio educa o deseduca, es necesario prestar atención a estos elementos. Además, desde el feminismo se visibilizan las consecuencias del aprendizaje patriarca al denunciar cómo determinados grupos de personas no ocupan los espacios, a veces directamente no están, otros quedan en los márgenes, a otras se les silencia o se les invisibiliza. Una vez más son aspectos que sirven para sustentar las estructuras de poder patriarcal. Un ejemplo de esto puede ser el uso de los patios escolares donde suele predominar el uso del juego de balón en el centro por parte de los niños quedando las niñas en las periferias del patio, o bien, en las salas de clase donde las voces protagonistas suelen ser de los varones imponiéndose a las opiniones de las mujeres u otros colectivos minoritarios. Las pedagogías feministas nos obligan a preguntarnos: ¿cómo ocupamos los espacios? ¿Quién aparece en primeras posiciones, quién toma la palabra, quién toma decisiones... y por el contrario, a quién se silencia, quién no aparece? ¿Usamos los espacios para reproducir o transformar las desigualdades?

- herstory, o historia de las mujeres: ¿lo que no se nombra no existe? ¿estamos las mujeres representadas en los contenidos, en la historia? ¿se conocen las aportaciones de la historia colectiva de las mujeres? Es interesante recuperar las palabras de Simone de Beauvoir (1949, p.48) al señalar que "la representación del mundo, como el mundo mismo, es operación de los hombres; ellos lo describen desde el punto de vista que les es propio, y que confunden con la verdad absoluta". Hablamos de conocer, de dar voz, de recuperar relatos 
olvidados y de escuchar la historia de las mujeres; historias cotidianas pero también grandes historias. Mujeres en las ciencias, en la literatura, en las artes, en los deportes... es necesario visibilizar, para tener referentes que rompan con los estereotipos normativos. Experiencias e investigaciones de pedagogías feministas recogen interesantes iniciativas que recopilan historias de vida de estas mujeres referentes en sus áreas, pero también recuperan el diálogo, la escucha intergeneracional, la construcción de narrativas y el aprendizaje de las ancestras como elemento esencial de la educación. A su vez denuncian la escasa presencia de mujeres en libros de texto, la invisibilización de modelos diversos y el predominio de imágenes estereotipadas de mujeres. Las propuestas que se hacen desde la pedagogía feminista consisten en: tener una actitud constante de revisión de los referentes, tanto masculinos como femeninos, que aparecen en libros, contenidos, actividades... así como visibilizar las contribuciones cotidianas de las mujeres más allá de las aportaciones de la academia, visibilizar acciones de subversión y de educación alternativa, nombrar a las mujeres y grupos minoritarios, construir educación emancipadora recuperando memoria, entre otras acciones.

- curriculum oculto, contenidos, ideologías, cultura educativa: se refiere a aquellas normativas, creencias, ideologías... oculta o no visible que configura el día a día de la educación. Se incluyen las creencias del profesorado, el reparto de liderazgo y cargos entre el profesorado, la brecha salarial y el techo de cristal entre el personal docente, la selección de contenidos y metodologías, la cultura organizacional, la ideología educativa, las construcciones de pensamiento, las experiencias personales, etc. que van configurando las relaciones de género y la inclusión de las diversidades. Es, por lo tanto, un elemento importante sobre el que tomar conciencia y visibilizar la reproducción de desigualdad que sustenta.

- lenguajes no sexistas: En relación a todo lo anterior es necesario atender también a las herramientas y canales de comunicación, los lenguajes que utilizamos y cómo los usamos. A través del lenguaje construimos imaginarios, cultura y narraciones; validamos identidades y exclusiones; nombramos nuestra realidad y a nosotras mismas (os); afianzamos esquemas mentales $\mathrm{y}$, socializamos y educamos. Las pedagogías feministas apuestan por usos del lenguaje diversos, reflejo Momento: diálogos em educação, E-ISSN 2316-3100, v. 28, n. 3, p. 350-365, set./dez, 2018. 
de sociedades dinámicas y complejas donde se nombre todas las realidades, se evite el uso de expresiones machistas, se visibilicen las diferencias, se desmonten ciertos calificativos y ofensas, y se amplíen las formas de comunicación más allá del lenguaje verbal tradicional.

- violencias machistas: sabemos que este apartado merece una dedicación particular, por su complejidad. Además, se considera que la violencia patriarcal es transversal a todas las dimensiones señaladas, es decir, el uso de espacios, la invisibilización de mujeres, los lenguajes machistas, el curriculum oculto... todas las desigualdades derivadas de ello son consecuencia de un sistema patriarcal que da sustento a las violencias machistas. Es por ello que la educación feminista no solo se hace eco de la realidad de feminicidios, sino que sensibiliza, analiza, crea conciencia crítica y construye acciones de resistencia ante las violencias. Hablamos de violencia física y evidente, pero también de esas violencias cotidianas, más sutiles, de los medios de comunicación, de las películas, de las canciones, de la publicidad, del acoso callejero, de la violencia familiar, del maltrato psicológicos, de los "micro" machismos, de la violencia económica, institucional, simbólica, etc. Las pedagogías feministas interpelan a la sociedad en su conjunto, como reproductora del sistema patriarcal, a tomar conciencia y posicionarse en contra de toda práctica, cultura y conducta que dé sustento a la violencia. Hablamos de una educación para la paz y la no violencia desde la emancipación de los grupos oprimidos/as y la deconstrucción de los sistemas de poder.

- masculinidades alternativas: tampoco se puede olvidar la necesidad de deconstruir las masculinidades hegemónicas y para ello es necesario, una vez más, tomar conciencia acerca de las bases estructurales que sustenta el patriarcado para reconstruir otras formas de ser hombres. Así, por ejemplo, es destacable como entre las pedagogías feministas se reclama un desarrollo educativo en torno a: el desarrollo emocional y la ética de los cuidados; la ruptura de lo dicotómico para poner en valor otras formas de ser y de hacer relacionadas con la sororidad, la escucha activa, la empatía y la colectividad; la deconstrucción del amor romántico y sus valores asociados para entender el mundo de las relaciones interpersonales desde la diversidad, el respeto a la integridad individual y el crecimiento conjunto; la expresión y gestión de emociones en la puesta de límites y el respeto a los mismos; el Momento: diálogos em educação, E-ISSN 2316-3100, v. 28, n. 3, p. 350-365, set./dez, 2018. 
aprendizaje de formas diversidad de sexualidad propias y ajenas, etc.

- metodologías de investigación-acción-participativa: relacionado con las metodologías de enseñanza y aprendizaje alternativas y rupturistas con las tendencias tradicionales de la enseñanza, las pedagogías feministas apuestan por procesos participativos y dialógicos desde la horizontalidad, la participación y el desarrollo comunitario. Es necesaria la toma de conciencia propia y colectiva del lugar que ocupamos en el mundo para hacernos partícipes activos del proceso educador, siendo este transformador. Recuperando palabras de Paulo Freire (1979), enseño porque busco, porque indagué, porque indago y me indago. Investigo para comprobar, comprobando intervengo, interviniendo educo y me educo. Investigo para conocer lo que aún no conozco y comunicar o anunciar la novedad.

A modo de síntesis y reflexión final cabe recordar que las pedagogías feministas ofrecen lugares comunes, compartidos, en los que situarnos para analizar, tomar conciencia y posicionarnos para deconstruir las desigualdades. Es dar cabida a las diferencias y ampliar los diálogos. Sus herramientas epistemológicas nos ayudan a repensar las prácticas educativas, sus sustentos culturales, los imaginarios dominantes y los mecanismos de normalización del sistema hegemónico. Hemos señalado aquellos relacionados con los lenguajes, los espacios, el curriculum, las metodologías, los contenidos, los estereotipos, entre otros.

Las pedagogías feministas proponen recuperar experiencias educativas centradas en estos principios y en diversos contextos que rompan con los espacios y tiempos tradicionales de la educación. Experiencias, teóricas y prácticas, que construyan conocimiento a partir de la narración no jerarquizada, tendiendo puentes entre el saber popular y el científico, entretejiendo pedagogías colectivas. Estas construcciones interpelan, desafían, subvierten y construyen nuevas formas de hacer y pensar la educación más allá de las imposiciones coloniales, jerárquicas, autoritarias, racistas y machistas. Supone una ruptura con el sistema patriarcal y sus enseñanzas, una revolución de las bases educativas.

Acabamos recordando a Korol (2016): “No es una pedagogía de profesoras y alumnas, sino de compañeras que soñamos juntas y pensamos juntas nuestras prácticas, que intentamos en el diálogo de saberes, crear nuevos conocimientos del mundo, ya que todo lo que fue creado hasta ahora, no alcanza para transformarlo", 


\section{REFERENCIAS}

Beauvoir, Simone de. (1949). El segundo sexo. España: Siglo XXI

Butler, Judith. (2001). El género en disputa. México: Ediciones Paidós..

Crenshaw, Kimberley. (1993). Demarginalizing the Intersection of Race and Sex: A Black Feminist Critique of Antidiscrimination Doctrine, Feminist Theory, and Antiracist Politics. En, D. Kelley Weisberg (Ed.) Feminist Legal Theory: Foundations. Philadelphia: Temple University Press, pp. 17-29.

Dewey, Jhon. (1970). Democracia y Educación. Buenos Aires: Losada.

Dussel, Enrique. (1999). Más allá del eurocentrismo: el sistema-mundo y los límites de la modernidad. En S. Castro-Gómez, O. Guardiola-Rivera, \& C.Millan, C. (Eds.), Pensar (en) los intersticios. Teoría y práctica de la crítica poscolonial. Bogotá: Instituto de Estudios Pensar-Universidad Javeriana, pp.147-161.

Federicci, Silvia, (2013). Revolución en punto cero. Trabajo doméstico, reproducción y luchas feministas. Madrid: Traficantes de sueños.

Freire, Paulo. (1969). Pedagogía como práctica de libertad. Madrid: Siglo XXI.

Galindo María. (2013). No se puede descolonizar sin despatriarcalizar. Teoría y propuesta de la despatriarcalización. La paz: Mujeres creando.

Galeano, Eduardo (2015). Mujeres. España: Siglo XXI.

Korol, Claudia. (2016). Feminismos populares. Pedagogías y políticas. Buenos Aires: América Libre.

Lagarde, Marcela. (2000). Género y feminismo. Desarrollo humano y democracia. Madrid, Horas y horas.

Martínez Martín, Irene (2016), Construcción de una pedagogía feminista para una ciudadanía transformadora y contra-hegemónica. Foro de Educación, 14(20), 129-151. doi: http://dx.doi. org/10.14516/fde.2016.014.020.008.

Martínez Martín, Irene y Ramírez, Gema. (2017). Despatriarcalizar y descolonizar la educación: Experiencias para una formación feminista del profesorado. Revista Internacional de Educación para la Justicia Social (RIEJS) 6, pp. 81-95. doi: 10.15366/riejs2017.6.2.005.

Mignolo, W. (2011). Geopolítica de la sensibilidad y del conocimiento Sobre (de) colonialidad, pensamiento fronterizo y desobediencia epistémica. Revista eipcp. Recuperado de: http://eipcp.net/transversal/0112/mignolo/es.

Millet, Kate. (1995). Política Sexual. Madrid: Cátedra 
Mohanty, Ch. (2008). Bajo los ojos de occidente. Academia feminista y discursos coloniales. En L. Suárez, \& R. Herrnández (Eds.) Descolonizando el Feminismo: teorías y prácticas desde los márgenes. Madrid: Cátedra, pp. 117-164.

Pérez Orozco, Amaia. (2007). Cadenas globales de cuidados. ONU.

http://mueveteporlaigualdad.org/publicaciones/cadenasglobalesdecuidado_orozco.pdf

Pérez, Amaia y del Río, Sira. (2002). La economía desde el feminismo: trabajos y cuidados. Recuperado de:

https://www.ecologistasenaccion.org/?p=13104\#outil_sommaire_8

Rubin, Gayle (1975). The traffic in women : notes on the political economy of sex, Reiter, R. (ed.). Toward and Anthropology of Women. New York, Monthly Review Press, pp. 157-210.

Van der Vleuten (1996) . Más allá de la intuición. Revista de docencia universitária, $1(2)$.

Varela, Nuria (2008). Feminismo para principiantes. España: Ediciones B

Walsh, C. (2013). Pedagogías decoloniales. Prácticas insurgentes de resistir, re-existir y re-vivir. Quito: Abya Yala. 